\title{
Validation of the Korean Version of the Gastroesophageal Reflux Disease Questionnaire for the Diagnosis of Gastroesophageal Reflux Disease
}

\author{
Eun Jeong Gong, ${ }^{1}$ Kee Wook Jung, ${ }^{2}$ Yang-Won Min, ${ }^{3}$ Kyoung Sup Hong, ${ }^{4}$ Hye-Kyung Jung, ${ }^{5}$ Hee Jung Son, ${ }^{3}$ Do Yeon Kim, ${ }^{2}$ \\ Jungbok Lee, ${ }^{6}$ and Oh Young Lee ${ }^{7 *}$ \\ ${ }^{I}$ Department of Internal Medicine, Gangneung Asan Hospital, University of Ulsan College of Medicine, Gangneung, Gangwon-do, Korea; \\ ${ }^{2}$ Department of Gastroenterology, University of Ulsan College of Medicine, Asan Medical Center, Seoul, Korea; ${ }^{3}$ Department of Gastroenterology, \\ Samsung Medical Center, Sungkyunkwan University School of Medicine, Seoul, Korea; ${ }^{4}$ Department of Internal Medicine, Seoul National \\ University College of Medicine, Seoul, Korea; ${ }^{5}$ Department of Internal Medicine, Ewha Womans University School of Medicine, Seoul, Korea; \\ ${ }^{6}$ Department of Clinical Epidemiology and Biostatistics, Asan Medical Center, Seoul, Korea; and ${ }^{7}$ Department of Internal Medicine, Hanyang \\ University School of Medicine, Seoul, Korea
}

\section{Background/Aims}

The Gastroesophageal Reflux Disease Questionnaire (GerdQ) has been developed and validated as a tool for the diagnosis of gastroesophageal reflux disease (GERD) in patients with gastrointestinal symptoms. However, the GerdQ and the cutoff value for determining GERD has not been validated in Korea.

\section{Methods}

Patients with symptoms suggestive of GERD were consecutively recruited. The Korean version of GerdQ was developed through a forward-backward translation process according to the cross-cultural adaptation method. Endoscopically documented esophagitis, abnormal results on 24-hour ambulatory $\mathrm{pH}$ recording with symptom association monitoring, or response to proton pump inhibitor treatment were used as diagnostic references for GERD. The reproducibility and test characteristics of the Korean version of GerdQ were assessed.

\section{Results}

A total of 149 patients with a median age of 55 years were analyzed. The intra-class correlation coefficient of 2 subsequently measured GerdQ scores was 0.651 (95\% Cl, 0.518-0.748). The cutoff value of 8 was found to have the highest sensitivity (64.9\%; $95 \% \mathrm{Cl}, 56.2-73.7)$ and specificity $(71.4 \% ; 95 \% \mathrm{Cl}, 56.5-86.4)$ for the diagnosis of GERD. The questionnaire had a high positive predictive value (88.1\%; $95 \% \mathrm{Cl}, 81.2-95.0)$, but a low negative predictive value (38.5\%; $95 \% \mathrm{Cl}, 26.2-50.3)$ for GERD. Any symptom improvement on proton pump inhibitor treatment showed a sensitivity of $93.0 \%(95 \% \mathrm{Cl}, 88.3-97.7)$ and a specificity of $48.6 \%(95 \%$ Cl, 32.0-65.1) for GERD.

\section{Conclusion}

The Korean version of GerdQ is a useful complementary tool in the diagnosis of GERD.

(J Neurogastroenterol Motil 2019;25:91-99)

Key Words

Gastroesophageal reflux; Surveys and questionnaires; Symptom assessment

Received: August 3, 2018 Revised: October 16, 2018 Accepted: October 25, 2018

(a) This is an Open Access article distributed under the terms of the Creative Commons Attribution Non-Commercial License (http://creativecommons. org/licenses/by-nc/4.0) which permits unrestricted non-commercial use, distribution, and reproduction in any medium, provided the original work is properly cited.

${ }^{*}$ Correspondence: Oh Young Lee, MD, PhD

Department of Internal Medicine, Hanyang University School of Medicine, 222-1 Wangsimni-ro, Seongdong-gu, Seoul 04763, Korea

Tel: +82-2-2290-8343, Fax: +82-2-2298-8314, E-mail: leeoy@hanyang.ac.kr

Eun Jeong Gong and Kee Wook Jung contributed equally to this work. 


\section{Introduction}

Gastroesophageal reflux disease (GERD) is a common condition characterized by symptoms or complications associated with reflux of gastric contents. ${ }^{1}$ Although the prevalence of GERD is relatively lower in Asia than in Western countries, the prevalence of GERD is increasing in Korea. ${ }^{2-4}$ As GERD is clinically diagnosed mainly on the basis of the presence of the cardinal symptoms of heartburn and acid regurgitation, the diagnosis of GERD remains challenging. ${ }^{1,5,6}$ Neither symptom-based evaluation nor objective measures including endoscopy and ambulatory $\mathrm{pH}$ monitoring are solely diagnostic for GERD. ${ }^{7}$ A short-course treatment trial with a proton pump inhibitor (PPI) is also used as a diagnostic method; however, it has shown only modest sensitivity and specificity. ${ }^{8,9}$

A number of symptom-based questionnaires and quality of life instruments have been developed to facilitate the diagnosis and management of GERD. ${ }^{9-11}$ However, most of these questionnaires were developed to assess symptom changes or the treatment outcome in epidemiological studies, rather than to make a diagnosis. Moreover, some of the questionnaires are composed of a wide variety of questions, requiring considerable time for patients to understand and complete, leading to their limited use in clinical practice.

The Gastroesophageal Reflux Disease Questionnaire (GerdQ) is a simple, 6-item, self-administered tool in which the frequency of the symptoms over the previous week is graded on a 4-point Likert scale. $^{9,12}$ The questionnaire was developed and validated primarily as a tool for untreated primary-care populations with upper gastrointestinal symptoms, and it has been found to be useful in guiding both diagnosis and management of GERD in Western countries. However, the Korean version of GerdQ has not yet been validated, and the ideal cutoff value for the diagnosis of GERD in the Korean population and its clinical implication have not been clearly defined. In this study, we aim to evaluate the validity of the Korean version of GerdQ.

\section{Materials and Methods}

\section{Subjects}

From December 2014 to January 2017, patients who had symptoms suggestive of GERD and visited 1 of 4 gastroenterology outpatient clinics (Asan Medical Center, Seoul National University Hospital, Samsung Medical Center, and Hanyang University Hospital) were recruited. Those with alarm symptoms such as progressive dysphagia, unintentional weight loss, or gastrointestinal bleeding were excluded. All enrolled patients provided written informed consent to participate in the study. The study protocol was approved by the institutional review board of each center (Registration No. 2014-1157, 1504-074-665, 2015-04-005, and 201606-001-003) and conducted in accordance with the Declaration of Helsinki and its amendments, as well as the Good Clinical Practice guidelines.

Patients were requested to answer the questionnaire, and the same questionnaire was administered again at least 1 week after the completion of the first questionnaire to evaluate the test-retest reliability. Endoscopic examination was performed according to the routines of each clinic, and the Los Angeles (LA) classification was used for grading reflux esophagitis. ${ }^{13}$ When there was no evidence of esophagitis on endoscopy, 24-hour pH monitoring was tried. All endoscopic evaluations and $\mathrm{pH}$ monitoring were performed by experienced and board-certified endoscopists. After the diagnostic investigations including endoscopy or esophageal manometry with 24-hour ambulatory $\mathrm{pH}$ monitoring, the patients returned to the outpatient clinic and a standard dose of PPI was prescribed on the basis of the results of upper endoscopy or $\mathrm{pH}$ testing. The response to PPI was asked through face-to-face interviews, and symptoms were assessed again by using the questionnaire at the end of the PPI treatment. When the patient did not visit the clinic, a telephone survey was conducted (Fig. 1).

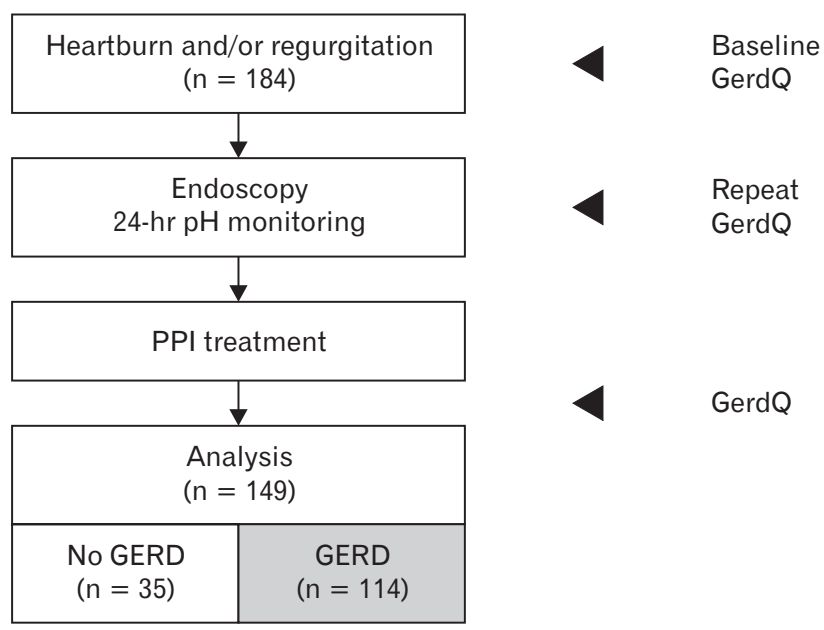

Figure 1. Flowchart of this study. GerdQ, Korean version of the Gastroesophageal Reflux Disease Questionnaire; PPI, proton pump inhibitor; GERD, gastroesophageal reflux disease. 


\section{Korean Gastroesophageal Reflux Disease Questionnaire}

The questionnaire comprises of 4 positive predictors of GERD (heartburn, regurgitation, sleep disturbance, and use of over-thecounter medication in addition to that prescribed) and 2 negative predictors of GERD (epigastric pain and nausea) (Table 1). All items were graded using a 4-grade scale (no symptoms, 1 day of symptoms, 2-3 days of symptoms, and 4-7 days of symptoms, over a 1-week recall period); scores ranging from 0 to 3 were applied for the positive predictors and from 3 to 0 for the negative predictors. The GerdQ score was calculated as the sum of these scores and ranged from 0 to 18 .

To develop the Korean version of GerdQ, translation was performed with authorization from the original author of GerdQ. The translation procedures included both forward and backward translation according to the cross-cultural adaptation method. The original English version of GerdQ was translated into Korean language by a Korean researcher who is aware of the objective of the questionnaire. Thereafter, 2 different bilingual professional translators, who had no knowledge about the questionnaire, translated it into English again. The backward-translated version was then compared with the original GerdQ to ensure that the meaning of the original questionnaire is retained in the Korean version. The wordings were discussed, and the questionnaire was modified accordingly. Items that seemed inaccurate or did not express the concept intended in the original version were re-translated by a second Korean researcher who was blind to the results of the previous translation. The Korean version of GerdQ was finalized after the resolution of any discrepancy and after proofreading by both the authors and translators.

\section{Criteria for the Diagnosis of Gastroesophageal Reflux Disease}

The diagnosis of GERD was made if at least one of the following criteria were met: (1) reflux esophagitis of LA classification A-D; (2) pathological findings on 24-hour $\mathrm{pH}$ monitoring, defined by fulfilling 1 of the following criteria: (i) $\mathrm{pH}<4.0$ for $\geq 4 \%$ of the time, or (ii) there is positive symptom association probability $(\geq$ 95\%) or positive symptom index ( $\geq 50 \%$ ) irrespective of the level of acid exposure; (3) positive response of reflux-related symptoms to PPI treatment, defined an answer of "yes" to the direct question of whether treatment had sufficiently controlled the symptoms after PPI treatment. ${ }^{14-16}$

\section{Statistical Methods}

For the estimation of the number of patients, the prevalence of GERD was assumed to be $60 \%$ in the study cohort. When the significance level (alpha) and the power of the test ( 1 - beta) were targeted at 0.05 and 0.9 , respectively, and the sensitivity and specificity both at $65 \%$, the sample size calculated using the one sample sensitivity and specificity power analysis was 155 . Considering a $15 \%$ dropout rate or loss to follow-up during the study period, it was estimated that a target enrollment of 180 subjects was needed.

The final version of the Korean GerdQ was tested on patients who visited the outpatient clinic, after which they provided consent. Responses on the 2 subsequent questionnaires were compared using kappa statistics to ensure that the responses given demonstrate an acceptable level of agreement (kappa > 0.4). Items in the Korean GerdQ that demonstrated poor agreement were reformatted as necessary, and a repeat backward translation was performed. Once the kappa value $>0.4$ has been achieved, concurrent validity testing was performed.

Table 1. The Gastroesophageal Reflux Disease Questionnaire (Adapted From Jones et al ${ }^{12}$ With Permission)

\begin{tabular}{|c|c|c|c|c|}
\hline \multirow{2}{*}{ Question } & \multicolumn{4}{|c|}{ Frequency score (points) for symptom } \\
\hline & 0 day & 1 day & 2-3 days & 4-7 days \\
\hline How often did you have a burning feeling behind your breastbone (heartburn)? & 0 & 1 & 2 & 3 \\
\hline $\begin{array}{l}\text { How often did you have stomach contents (liquid or food) moving upwards to your throat or mouth } \\
\text { (regurgitation)? }\end{array}$ & 0 & 1 & 2 & 3 \\
\hline How often did you have pain in the center of the upper stomach? & 3 & 2 & 1 & 0 \\
\hline How often did you have nausea? & 3 & 2 & 1 & 0 \\
\hline $\begin{array}{l}\text { How often did you have difficulty getting a good night's sleep because of your heartburn and/ } \\
\text { or regurgitation? }\end{array}$ & 0 & 1 & 2 & 3 \\
\hline $\begin{array}{l}\text { How often did you take additional medication for your heartburn and/or regurgitation, other than } \\
\text { what the physician told you to take? }\end{array}$ & 0 & 1 & 2 & 3 \\
\hline
\end{tabular}


The test-retest reproducibility was assessed by calculating the intra-class correlation coefficient between the scores of the first and the repeated responses to the questionnaire, which were obtained 1 week apart. A receiver operating characteristic curve was used to determine the optimum cutoff value. The cutoff score was selected to maximize the tradeoff between sensitivity and specificity. The sensitivity, specificity, positive and negative predictive value, and area under the receiver operating characteristic curve were estimated. All statistical analyses were performed using SPSS version 21.0 (IBM Corp, Armonk, NY, USA), and $P$-values $<0.05$ were considered statistically significant.

\section{Results}

A total of 184 consecutive patients were screened, and, finally, 149 patients who fully completed the questionnaire and the diagnostic procedures were analyzed. The baseline characteristics of the study patients are summarized in Table 2 . The median age was 55 years and $34.9 \%$ were men. Of the patients who underwent endoscopy, $12.3 \%$ were found to have reflux esophagitis, and $11.6 \%$ had hiatal hernia. Table 3 shows the proportions of patients who reported each symptom included in the questionnaire. Considering the typical symptoms, 106 patients $(71.1 \%)$ reported heartburn and $111(74.5 \%)$ complained of regurgitation. Sleep disorder was reported by $45.0 \%$ of the patients, whereas epigastric pain and nausea were present in more than two-thirds of all patients.

Among the study patients with reflux symptoms, 114 patients (76.5\%) fulfilled the criteria for the diagnosis of GERD. Among 131 patients who did not have erosive reflux esophagitis, 13 underwent $\mathrm{pH}$ monitoring and 5 showed pathologic acid reflux. In 126 patients having no reflux esophagitis or pathologic acid reflux, 91 patients had positive symptom response to PPI.

The distribution of the GerdQ score and the number of patients with GERD are shown in Figure 2. The median baseline
GerdQ score was 8 (range, 3-15), and the proportion of patients who had reflux-related symptoms $\geq 2$ days during the last week increased as the GerdQ score increased (Fig. 3). The intra-class correlation coefficient of two subsequently measured GerdQ scores was 0.651 (95\% CI, 0.518-0.748).

Based on the receiver operating characteristic curve, a GerdQ score of $\geq 8$ showed a sensitivity of $64.9 \%$ (95\% CI, 56.2-73.7) and a specificity of $71.4 \%$ (95\% CI, 56.5-86.4) for the diagnosis of GERD with an area under the curve of 0.741 (standard error 0.042 )

Table 2. Baseline Characteristics of the Study Population

\begin{tabular}{lc}
\multicolumn{1}{c}{ Variables } & $\mathrm{N}=149$ \\
\hline Age $(\mathrm{yr})$ & $55.0(19.0-79.0)$ \\
Male & $52(34.9)$ \\
Body mass index $\left(\mathrm{kg} / \mathrm{m}^{2}\right)$ & $23.1(15.6-35.1)$ \\
Symptom score at baseline & \\
Median GerdQ score & $8(3-15)$ \\
GerdQ score $\geq 8$ & $84(56.4)$ \\
GerdQ score $\geq 9$ & $57(38.3)$ \\
GerdQ score $\geq 10$ & $43(28.9)$ \\
Endoscopic findings (n $=146)$ & \\
Reflux esophagitis & $18(12.3)$ \\
LA grade A & $12(8.2)$ \\
LA grade B & $5(3.4)$ \\
LA grade C & $1(0.7)$ \\
LA grade D & 0 \\
Hiatal hernia & $17(11.6)$ \\
Peptic ulcer & $1(0.7)$ \\
Stricture & 0 \\
Barrett's esophagus & 0 \\
24-hr ambulatory pH monitoring & $13(8.7)$ \\
Patients who underwent the test & $5(3.4)$ \\
Patients with pathological results & \\
\hline
\end{tabular}

$\overline{\text { GerdQ, Gastroesophageal Reflux Disease Questionnaire; LA, Los Angeles }}$ classification.

Data represent number $(\%)$ or median (range).

Table 3. Distribution of Patients Who Reported Positive Symptoms Included in the Gastroesophageal Reflux Disease Questionnaire

\begin{tabular}{lcccc}
\hline & \multicolumn{4}{c}{ Frequency of symptom } \\
\cline { 2 - 4 } Symptom assessed & 0 day & 1 day & $2-3$ days & $4-7$ days \\
\hline Heartburn & $43(28.9)$ & $18(12.1)$ & $39(26.2)$ & $49(32.9)$ \\
Regurgitation & $38(25.5)$ & $27(18.1)$ & $40(26.8)$ & $44(29.5)$ \\
Epigastric pain & $33(22.1)$ & $41(27.5)$ & $17(11.4)$ & $58(38.9)$ \\
Nausea & $15(10.1)$ & $26(22.1)$ & $24(16.1)$ & $80(53.7)$ \\
Sleep disorder & $82(55.0)$ & $10(6.7)$ & $9(6.0)$ & $17(11.4)$ \\
Use of medications & $120(80.5)$ & & $10(6.7)$ \\
\hline
\end{tabular}

Data represent number (\%). 
A

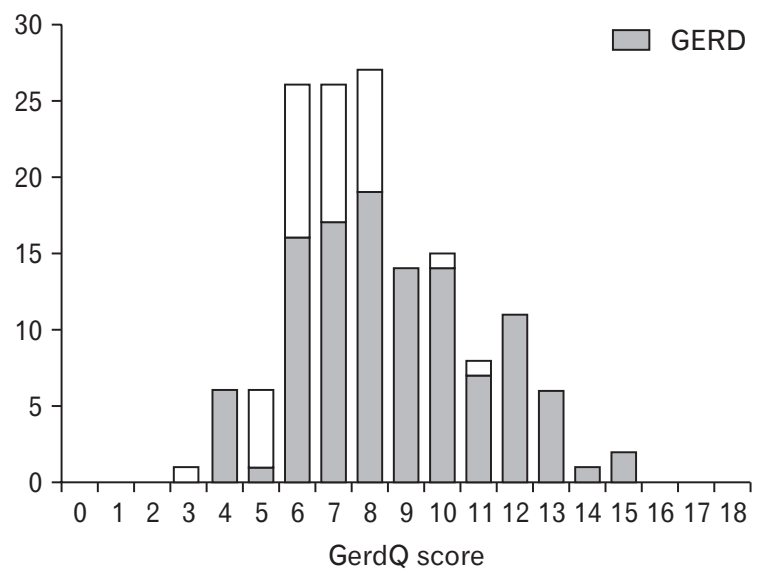

B

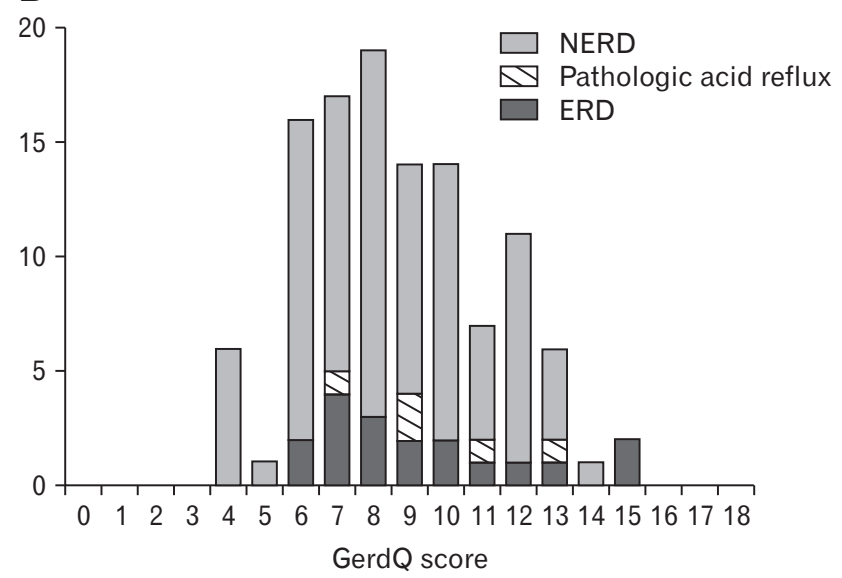

Figure 2. The distribution of Korean version of the Gastroesophageal Reflux Disease Questionnaire (GerdQ) score (sum of 6 items, 0-18) at baseline. (A) The proportion of patients with gastroesophageal reflux disease (GERD) by GerdQ score. (B) Characteristics of patients with GERD according to the diagnostic criteria. NERD, non-erosive reflux esophagitis; ERD, erosive reflux esophagitis.

(Table 4 and Fig. 4). The Korean version of GerdQ showed a high positive predictive value $(88.1 \%$; $95 \% \mathrm{CI}, 81.2-95.0)$ but a low negative predictive value (38.5\%; 95\% CI, 26.2-50.3) for GERD.

All patients received PPI treatment, and the median GerdQ score was 6 (range, 2-12) after PPI treatment. The median decrease in the GerdQ score was 2, which was significantly different between patients with and without GERD (median 3 and $1, P<0.001$ ). A total of 107 patients (71.8\%) reported subjective improvement in their symptoms, and the decrease in GerdQ score could predict GERD with a sensitivity of $93.0 \%$ (95\% CI, 88.3-97.7) and specificity of $48.6 \%$ (95\% CI, 32.0-65.1) (Table 5).

\section{Discussion}

In this study, we investigated the validity of the Korean version of GerdQ for the diagnosis of GERD in patients with upper gastrointestinal symptoms. With a cutoff value of 8 , the Korean version of GerdQ was found to have a sensitivity of $64.9 \%$ and a specificity of $71.4 \%$ for the diagnosis of GERD. This result suggests that the GerdQ could be adapted to the Korean population while maintaining the high efficacy of the symptom-based diagnosis of GERD.

GERD is a complex clinical condition with a wide spectrum of clinical presentations. Heartburn and regurgitation are considered typical symptoms of GERD, and the current guideline recommends symptom-based diagnosis and empirical treatment unless alarm symptoms mandate prompt endoscopy. ${ }^{5}$ These typical symptoms are highly specific for GERD, but have low sensitivity for the diagnosis of GERD. ${ }^{7,17}$ Patients often present symptom clusters, and heartburn or regurgitation was the most troublesome symptom in only $49 \%$ of the patients with GERD. ${ }^{9}$ Furthermore, the presence of atypical or extraesophageal symptoms makes accurate diagnosis challenging. Therapeutic trials with PPI have been widely performed; however, they have shown limited value in terms of diagnostic precision. ${ }^{8,18}$ Even objective measures including endoscopy and 24-hour $\mathrm{pH}$ monitoring are not sufficiently sensitive as diagnostic tools. ${ }^{19,20}$ In Korea, as most patients with reflux symptoms show no evidence of esophagitis on endoscopy, a symptom-based approach remains a pivotal step in the diagnosis and management of GERD. ${ }^{3,21}$

An accurate diagnosis of GERD is important because untreated or persistent symptoms may negatively affect the patients' healthrelated quality of life. ${ }^{4,22}$ Moreover, suboptimal treatment may result in unnecessary clinical investigations and excessive healthcare costs. There have been attempts to develop structured questionnaires to improve the diagnosis and management of GERD. However, many of those questionnaires are complex, containing a huge number of items about various symptoms. GerdQ is a simple and readily available, self-administered, 6-item questionnaire that was developed as a tool to facilitate symptom-based diagnosis and evaluation of treatment response in patients with GERD. ${ }^{12,23}$ This questionnaire would not only assist in making the diagnosis but would also provide a structured treatment approach, resulting in reduced healthcare costs. ${ }^{24,25}$ In a previous validation study, GerdQ had a sensitivity of $65 \%$ and specificity of $64 \%$ for the diagnosis of GERD when using the cutoff score of $9 .^{23}$ The Korean version of GerdQ was found to have similar diagnostic performance to that in 


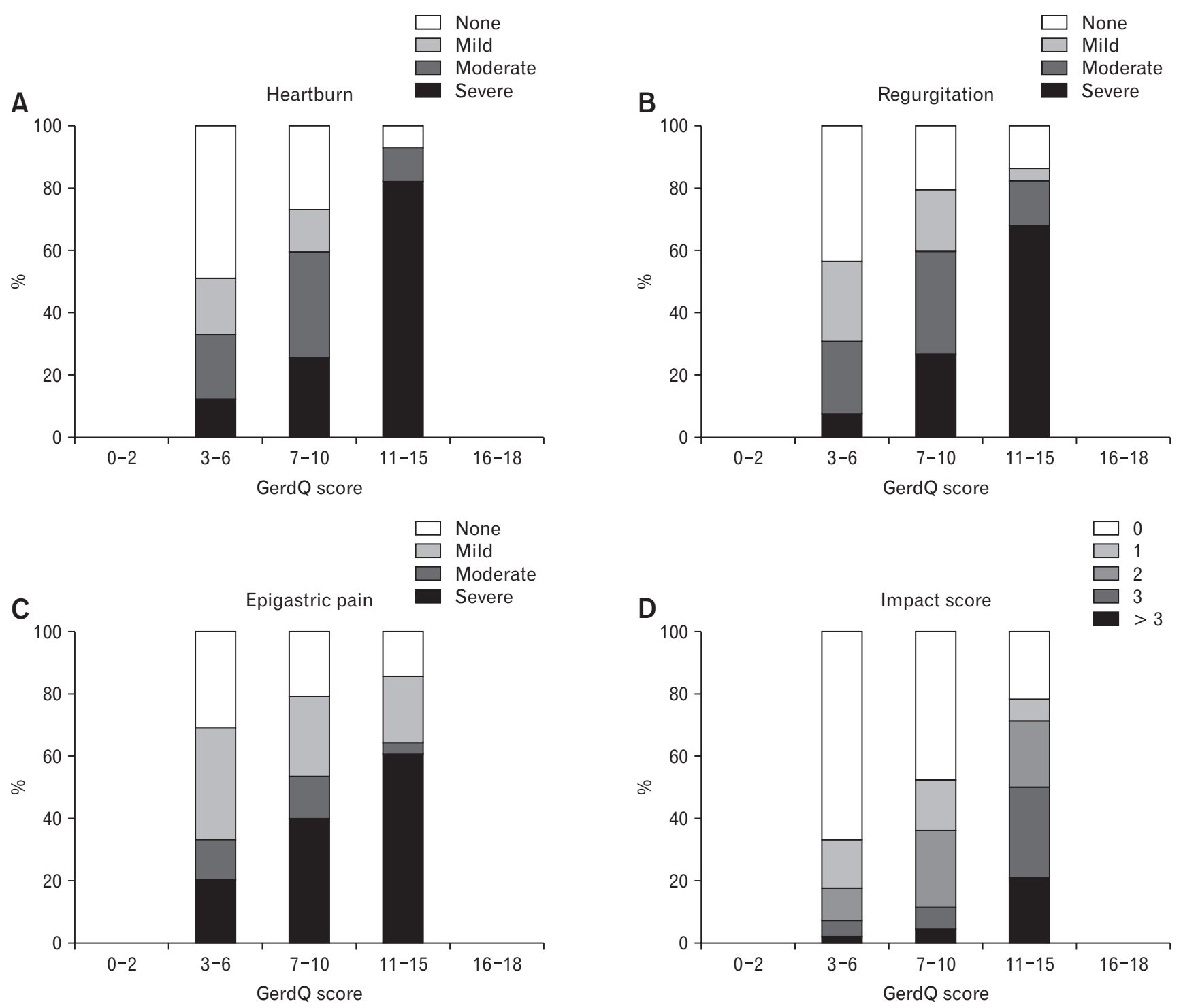

Figure 3. Distribution of patients by Korean version of the Gastroesophageal Reflux Disease Questionnaire (GerdQ) score and baseline symptom frequency: (A) heartburn, (B) regurgitation, (C) epigastric pain, and (D) impact score comprising the summed score of sleep disorder and medication use.

the previous study, with the cutoff score of 8 . Whereas more than half of the patients had a GerdQ score of $\geq 10$ in a previous Western study, ${ }^{23}$ only $28.9 \%$ of patients showed a GerdQ score of $\geq$ 10 in the present study. In addition, there was no patient with LA grade D esophagitis or a GerdQ score of $>15$. These differences in symptom severity might have contributed to the relatively lower cutoff score in the Korean population than that reported in Western studies.

The ideal instrument for symptom assessment should be patient assessed, easy to understand, practical, and valid in different languages. ${ }^{26}$ As both the perception and expression of symptoms are different according to individual patients, symptom-based assessment can often lead to misinterpretation. Linguistic and cultural differences can also influence the understanding of self-administered questionnaires. In particular, it is often difficult to describe the nature of heartburn properly without losing relevance to the symptoms, as there is no precise translation of the word "heartburn" in Korean. A previous study showed that the symptom description that was most commonly chosen by patients with a "burning feeling" was pain or discomfort in the stomach. ${ }^{27}$ In addition to day-today variation in symptom occurrence and recall bias, inconsistency in the patients' perception or expression of symptoms might have 
Table 4. Test Characteristics of the Gastroesophageal Reflux Disease Questionnaire Score in the Diagnosis of Gastroesophageal Reflux Disease

\begin{tabular}{ccccc}
\hline $\begin{array}{c}\text { Cutoff } \\
\text { score }\end{array}$ & $\begin{array}{c}\text { Sensitivity } \\
\%(95 \% \mathrm{CI})\end{array}$ & $\begin{array}{c}\text { Specificity } \\
\%(95 \% \mathrm{CI})\end{array}$ & $\begin{array}{c}\text { PPV } \\
\%(95 \% \mathrm{CI})\end{array}$ & $\begin{array}{c}\mathrm{NPV} \\
\%(95 \% \mathrm{CI})\end{array}$ \\
\hline 7 & 79.8 & 45.7 & 82.7 & 41.0 \\
& $(72.5-87.2)$ & $(29.2-62.2)$ & $(75.7-89.8)$ & $(25.6-56.5)$ \\
8 & 64.9 & 71.4 & 88.1 & 38.5 \\
& $(56.2-73.7)$ & $(56.5-86.4)$ & $(81.2-95.0)$ & $(26.6-50.3)$ \\
9 & 48.2 & 94.3 & 95.5 & 35.9 \\
& $(39.1-57.4)$ & $(86.6-102.0)$ & $(91.7-101.3)$ & $(26.1-45.7)$ \\
\hline
\end{tabular}

$\mathrm{NPV}$, negative predictive value; PPV, positive predictive value.

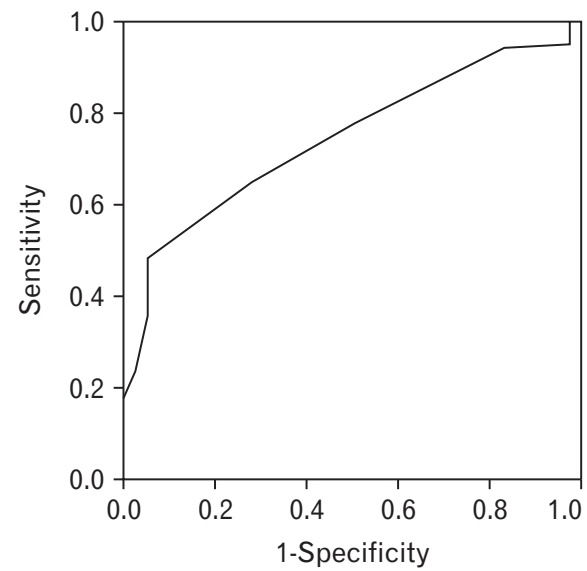

Figure 4. Receiver operating characteristic curve of the Korean version of the Gastroesophageal Reflux Disease Questionnaire for the diagnosis of gastroesophageal reflux disease. The sensitivity and specificity at the cutoff value of 8 were $64.9 \%$ and $71.4 \%$, respectively, and the area under the receiver operating characteristic curve was 0.741 (SE 0.042).

led to a suboptimal level of intra-class correlation coefficient in this study.

The symptom overlap between GERD and functional disorders such as functional dyspepsia or irritable bowel syndrome has been reported to be substantial, suggesting a possible common pathophysiology such as altered visceral sensitivity. ${ }^{28}$ About $50 \%$ of Korean patients with GERD also complained of dyspepsia. ${ }^{4}$ Conversely, $23 \%$ of patients with functional dyspepsia showed pathological acid reflux on 24-hour $\mathrm{pH}$ monitoring, suggesting that a certain subgroup of patients with functional dyspepsia may respond to PPI treatment. ${ }^{29}$ Notably, more than two-thirds of patients complained of epigastric pain or nausea at least once a week in the present study, suggesting that our study population might have consisted of heterogeneous patients, including those with overlap syndrome.
Table 5. Test Characteristics of the Response to Proton Pump Inhibitor Treatment in the Diagnosis of Gastroesophageal Reflux Disease

\begin{tabular}{ccccc}
\hline $\begin{array}{c}\text { Decrease } \\
\text { in GerdQ } \\
\text { score }\end{array}$ & $\begin{array}{c}\text { Sensitivity } \\
\%(95 \% \mathrm{CI})\end{array}$ & $\begin{array}{c}\text { Specificity } \\
\%(95 \% \mathrm{CI})\end{array}$ & $\begin{array}{c}\text { PPV } \\
\%(95 \% \mathrm{CI})\end{array}$ & $\begin{array}{c}\text { NPV } \\
(95 \% \mathrm{CI})\end{array}$ \\
\hline$\geq 1$ & 93.0 & 48.6 & 85.5 & 68.0 \\
& $(88.3-97.7)$ & $(32.0-65.1)$ & $(79.3-91.7)$ & $(49.7-86.3)$ \\
$\geq 2$ & 78.9 & 80.0 & 92.8 & 53.8 \\
& $(71.5-86.4)$ & $(66.7-93.3)$ & $(87.6-98.0)$ & $(40.3-67.4)$ \\
$\geq 3$ & 56.1 & 94.3 & 97.0 & 39.8 \\
& $(47.0-65.2)$ & $(86.6-102.0)$ & $(92.8-101.1)$ & $(29.2-50.3)$ \\
\hline
\end{tabular}

$\mathrm{NPV}$, negative predictive value; PPV, positive predictive value; GerdQ, Gastroesophageal Reflux Disease Questionnaire.

Our study has several limitations. First, as it was performed in tertiary-care centers, the patients in this study may not be representative of the general population. Second, the spectrum of patients meeting the diagnostic criteria for GERD was broad, comprising those with endoscopic esophagitis or abnormal acid reflux as well as those who showed a positive response to PPI treatment in the absence of an objective evidence or acid reflux. In the present study, we used the GERD criteria based on the previously published guideline. ${ }^{6,30}$ The recently published Lyon consensus suggested that an acid exposure time of $>6 \%$ on $\mathrm{pH}$ monitoring and LA grade $\mathrm{C}$ or $\mathrm{D}$ esophagitis could be the conclusive determinants of pathologic reflux. ${ }^{31}$ However, in the Korean population, GERD tended to be less severe and uncomplicated, being either non-erosive reflux disease or mostly LA grade A or B. ${ }^{32}$ Moreover, the previously published normal value of acid exposure time on 24-hour $\mathrm{pH}$ testing for Korean patients was $3.7 \% .^{33}$ Therefore, we tried to apply the criteria that reflect the characteristics of Korean patients in the diagnosis of GERD. Another possible limitation of our study is that we included only a few patients who did not have GERD.

In conclusion, the Korean version of GerdQ is a useful complementary tool for the diagnosis of GERD, based on its the simplicity and straightforward translation. There is no gold standard in the diagnosis of GERD, and the presence or absence of specific symptoms is not sufficient to make or disregard the diagnosis. Rather, a comprehensive approach encompassing symptoms as well as objective measures are desirable to reach the accurate diagnosis. Further validation of this instrument may be beneficial to confirm its robustness for measuring the treatment response in GERD.

Financial support: This work was supported by AstraZeneca.

\section{Conflicts of interest: None.}


Author contributions: Study conception, design, and supervision: Kee Wook Jung, Hye-Kyung Jung, and Oh Young Lee; patient recruitment, data collection, and data analysis and interpretation: Kee Wook Jung, Yang-Won Min, and Kyoung Sup Hong; writing of the first draft: Eun Jeong Gong and Kee Wook Jung; review of medical records, data collection, and data analysis and interpretation: Eun Jeong Gong, Hee Jung Son, and Do Yeon Kim; review of statistic data: Jungbok Lee; and all authors were involved in critical revision of the paper.

\section{References}

1. Vakil N, van Zanten SV, Kahrilas P, Dent J, Jones R; Global Consensus Group. The montreal definition and classification of gastroesophageal reflux disease: a global evidence-based consensus. Am J Gastroenterol 2006;101:1900-1920.

2. El-Serag HB, Sweet S, Winchester CC, Dent J. Update on the epidemiology of gastro-oesophageal reflux disease: a systematic review. Gut 2014;63:871-880.

3. Kim KM, Cho YK, Bae SJ, et al. Prevalence of gastroesophageal reflux disease in Korea and associated health-care utilization: a national population-based study. J Gastroenterol Hepatol 2012;27:741-745.

4. Min BH, Huh KC, Jung HK, et al. Prevalence of uninvestigated dyspepsia and gastroesophageal reflux disease in Korea: a population-based study using the Rome III criteria. Dig Dis Sci 2014;59:2721-2729.

5. Katz PO, Gerson LB, Vela MF. Guidelines for the diagnosis and management of gastroesophageal reflux disease. Am J Gastroenterol 2013;108:308-328.

6. Jung HK, Hong SJ, Jo YJ, et al. [Updated guidelines 2012 for gastroesophageal reflux disease.] Korean J Gastroenterol 2012;60:195-218. [Korean]

7. Klauser AG, Schindlbeck NE, Müller-Lissner SA. Symptoms in gastrooesophageal reflux disease. Lancet 1990;335:205-208.

8. Cho YK, Choi MG, Lim CH, et al. Diagnostic value of the PPI test for detection of GERD in Korean patients and factors associated with PPI responsiveness. Scand J Gastroenterol 2010;45:533-539.

9. Dent J, Vakil N, Jones R, et al. Accuracy of the diagnosis of GORD by questionnaire, physicians and a trial of proton pump inhibitor treatment: the diamond study. Gut 2010;59:714-721.

10. Shaw M, Dent J, Beebe T, et al. The reflux disease questionnaire: a measure for assessment of treatment response in clinical trials. Health Qual Life Outcomes 2008;6:31.

11. Rubin G, Uebel P, Brimo-Hayek A, Hey KH, Doerfler H, Heading $\mathrm{RC}$. Validation of a brief symptom questionnaire (ReQuest in Practice) for patients with gastro-oesophageal reflux disease. Aliment Pharmacol Ther 2008;27:846-851.

12. Jones R, Junghard O, Dent J, et al. Development of the GerdQ, a tool for the diagnosis and management of gastro-oesophageal reflux disease in primary care. Aliment Pharmacol Ther 2009;30:1030-1038.

13. Lundell LR, Dent J, Bennett JR, et al. Endoscopic assessment of oe- sophagitis: clinical and functional correlates and further validation of the Los Angeles classification. Gut 1999;45:172-180.

14. Cho YK, Choi MG, Lim CH, et al. Impaired esophageal bolus transit in patients with gastroesophageal reflux disease and abnormal esophageal acid exposure. Gut Liver 2012;6:440-445.

15. Kim BJ, Choi SC, Kim JJ, Rhee JC, Rhee PL. Pathological bolus exposure plays a significant role in eliciting non-cardiac chest pain. J Gastroenterol Hepatol 2010;25:1855-1860.

16. Vela MF. Diagnostic work-up of GERD. Gastrointest Endosc Clin N Am 2014;24:655-666.

17. Moayyedi P, Talley NJ, Fennerty MB, Vakil N. Can the clinical history distinguish between organic and functional dyspepsia? JAMA 2006;295:1566-1576.

18. Bytzer P, Jones R, Vakil N, et al. Limited ability of the proton-pump inhibitor test to identify patients with gastroesophageal reflux disease. Clin Gastroenterol Hepatol 2012;10:1360-1366.

19. Sifrim D, Castell D, Dent J, Kahrilas PJ. Gastro-oesophageal reflux monitoring: review and consensus report on detection and definitions of acid, non-acid, and gas reflux. Gut 2004;53:1024-1031.

20. Mäntynen T, Färkkilä M, Kunnamo I, Mecklin JP, Juhola M, Voutilainen M. The impact of upper GI endoscopy referral volume on the diagnosis of gastroesophageal reflux disease and its complications: a 1-year cross-sectional study in a referral area with 260,000 inhabitants. Am J Gastroenterol 2002;97:2524-2529.

21. Kim N, Lee SW, Cho SI, et al. The prevalence of and risk factors for erosive oesophagitis and non-erosive reflux disease: a nationwide multicentre prospective study in Korea. Aliment Pharmacol Ther 2008;27:173-185.

22. Tack J, Becher A, Mulligan C, Johnson DA. Systematic review: the burden of disruptive gastro-oesophageal reflux disease on health-related quality of life. Aliment Pharmacol Ther 2012;35:1257-1266.

23. Jonasson C, Wernersson B, Hoff DA, Hatlebakk JG. Validation of the GerdQ questionnaire for the diagnosis of gastro-oesophageal reflux disease. Aliment Pharmacol Ther 2013;37:564-572.

24. Jonasson C, Moum B, Bang C, Andersen KR, Hatlebakk JG. Randomised clinical trial: a comparison between a GerdQ-based algorithm and an endoscopy-based approach for the diagnosis and initial treatment of GERD. Aliment Pharmacol Ther 2012;35:1290-1300.

25. Ponce J, Garrigues V, Agréus L, et al. Structured management strategy based on the gastro-oesophageal reflux disease (GERD) questionnaire (GerdQ) vs. usual primary care for GERD: pooled analysis of five cluster-randomised European studies. Int J Clin Pract 2012;66:897-905.

26. Stanghellini V, Armstrong D, Monnikes H, Bardhan KD. Systematic review: do we need a new gastro-oesophageal reflux disease questionnaire? Aliment Pharmacol Ther 2004;19:463-479.

27. Carlsson R, Dent J, Bolling-Sternevald E, et al. The usefulness of a structured questionnaire in the assessment of symptomatic gastroesophageal reflux disease. Scand J Gastroenterol 1998;33:1023-1029.

28. Lee SY, Lee KJ, Kim SJ, Cho SW. Prevalence and risk factors for overlaps between gastroesophageal reflux disease, dyspepsia, and irritable bowel syndrome: a population-based study. Digestion 2009;79:196-201.

29. Tack J, Caenepeel P, Arts J, Lee KJ, Sifrim D, Janssens J. Prevalence of acid reflux in functional dyspepsia and its association with symptom pro- 
file. Gut 2005;54:1370-1376.

30. Vaezi MF, Sifrim D. Assessing old and new diagnostic tests for gastroesophageal reflux disease. Gastroenterology 2018;154:289-301.

31. Gyawali CP, Kahrilas PJ, Savarino E, et al. Modern diagnosis of GERD: the Lyon consensus. Gut 2018;67:1351-1362.

32. Shim KN, Hong SJ, Sung JK, et al. Clinical spectrum of reflux esopha- gitis among 25,536 Koreans who underwent a health check-up: a nationwide multicenter prospective, endoscopy-based study. J Clin Gastroenterol 2009;43:632-638.

33. Kim GH, Huh KC, Lee YC, et al. Normal ambulatory 24-hour esophageal $\mathrm{pH}$ values in Koreans -A multicenter study. J Korean Med Sci 2008;23:954-958. 